\title{
Politische Bildung auf Sekundar- stufe I. Erscheinungsformen und Herausforderungen am Beispiel von Unterrichtssequenzen zu «Europa - EU - Schweiz»
}

\author{
Julia Thyroff, Jan Scheller, Claudia Schneider und Monika Waldis
}

Fachhochschule Nordwestschweiz FHNW ${ }^{1}$

Mit Einführung des Lehrplans 21 stehen Geschichtslehrpersonen künftig vor der Herausforderung, auf Sekundarstufe I auch Politische Bildung zu unterrichten - ein Fach, das jedoch über ganz eigene Prinzipien, Perspektiven, Konzepte und Kompetenzen verfügt. Inwiefern gelingt es Lehrpersonen aktuell, diese genuin politische Fachlichkeit zu realisieren? Im Text stellen wir Ergebnisse unserer Unterrichtsanalysen vor, die wir anhand von acht Doppellektionen zum Gegenstand "Europa - EU - Schweiz», durchgeführt von vier Lehrpersonen, gewonnen haben. Wir fokussieren dabei auf zwei Aspekte: a) Inwiefern wird von den Lehrpersonen auf der Gegenstandsebene politische Fachlichkeit realisiert und b) inwieweit werden in den formulierten Arbeitsaufträgen politische Kompetenzen adressiert?

\section{Einleitung}

Mit Einführung des neuen Lehrplans 21 für die obligatorische Schule in der deutschsprachigen Schweiz erhält die Politische Bildung einen neuen Stellenwert: Sie wird einerseits als fächerübergreifendes Prinzip, andererseits als fester Bestandteil des Fachbereichs «Räume, Zeiten, Gesellschaften» auf Sekundarstufe I verankert und dort unter anderem von Geschichtslehrpersonen unterrichtet. Die bislang schwache Berücksichtigung Politischer Bildung in Ausbildungsgängen für Lehrpersonen sowie Befunde aus empirischen Studien (z. B. Allenspach, 2014; Bürgler \& Hodel, 2012; Waldis, Schneider, Hedinger, \& Thyroff, 2017) lassen allerdings annehmen, dass Lehrpersonen über einen begrenzten fachlichen Hintergrund und geringe fachdidaktische Kenntnisse verfügen und somit Politische Bildung trotz neuer formaler Festschreibung marginalisiert werden könnte. Im vom Schweizerischen Nationalfonds (SNF) geförderten Forschungsprojekt «Politische Bildung im fächerübergreifenden Unterricht mit Geschichte auf Sekundarstufe I» erkunden wir, wie unter den genannten Rahmenbedingungen Politische Bildung realisiert wird. Inwiefern gelingt es 
Geschichtslehrpersonen, genuine fachliche und fachdidaktische Anliegen Politischer Bildung umzusetzen? Zu diesen Anliegen zählen wir etwa die Einnahme einer politischen Perspektive auf Gegenstandsebene oder die Formulierung von Aufträgen, die politische Kompetenzen der Schüler*innen fördern sollen.

Im vorliegenden Artikel analysieren wir explorativ den Unterricht vierer Lehrpersonen, die "Europa» (z. B. europäische Institutionen) und das Verhältnis Europa-Schweiz behandelten und fragen: 1) Inwiefern wird auf der Gegenstandsebene der Blick auf das Politische gerichtet, also geraten bei der Thematisierung von Europa bzw. der EU Interessenvielfalt und damit verbundene konfliktive Aushandlungsprozesse in den Blick und wird dem Prinzip der Kontroversität Rechnung getragen? 2) Inwiefern werden durch die Aufgabenstellungen Fähigkeiten adressiert, die dem Erwerb politischer Kompetenzen dienlich sind? Nachfolgend schildern wir für beide Bereiche je gesondert die theoretische und empirische Ausgangslage (Kapitel 2 und 3), bevor wir dann unser methodisches Vorgehen und Ergebnisse darstellen (Kapitel 4, 5 und 6).

\section{Politische Fachlichkeit zum Gegen- stand Europa: Theoretische \& empirische Ausgangs lage ${ }^{2}$}

\section{Theoretische überlegungen}

Der Gegenstand «Europa» und die in Bezug auf ihn formulierten Anliegen innerhalb der Politikdidaktik sind durch eine ausgesprochene Mehrschichtigkeit und Unübersichtlichkeit gekennzeichnet. Eine Möglichkeit der Systematisierung ist die in ein Lernen bzw. eine Bildung in Europa, über Europa und für Europa (z. B. Rappenglück, 2004) - unter Verwendung einer Dreiteilung, wie diese ähnlich auch für die Menschenrechtsbildung gebräuchlich ist (z. B. Heldt, 2018).

Für uns ist hier das Lernen über Europa von Interesse, beziehungsweise die Frage, wie Lehrpersonen dieses angebotsseitig gestalten. Die Ausgangslage für Unterricht über Europa ist dabei freilich komplex: Zwar ist die Schweiz nicht Mitglied der EU, aber über Bilaterale Verträge mit dieser verbunden, Mitglied unter anderem des Europarats und der OECD. Und auch jenseits der institutionellen Ebene ist die Schweiz auf mannigfaltige Weise (geographisch, wirtschaftlich u.v.m.) Teil Europas.

Inwiefern gelingt es Lehrpersonen, in diesem komplexen Gefüge eine genuin politische Perspektive auf Europa einzunehmen? Unter Verwendung einer in der deutschsprachigen Politikdidaktik gebräuchlichen Definition fassen wir das Politische als Feld der «Regelung von grundlegenden Fragen und Problemen des gesamtgesellschaftlichen Zusammenlebens» (Gesellschaft für Politikdidaktik und politische Jugend- und Erwachsenenbildung [GPJE], 2004, S. 10).

Hierbei können prinzipiell vier Dimensionen zum Tragen kommen: zunächst «Politik im engeren Sinn» als "konfliktiver, konflikthafter und demokratischer 
Prozess zur Herstellung verbindlicher Entscheidungen», ergänzt um "wirtschaftliche Fragen und Probleme», "Fragen und Probleme des gesellschaftlichen Zusammenlebens» sowie «rechtliche Fragen und Probleme» (GPJE, 2004, S. 10 f.). In jedem Fall kommt dem Prinzip der Kontroversität (Grammes, 2014) konstitutive Bedeutung für die Auseinandersetzung mit dem Politischen zu.

Gerade beim Gegenstand Europa können Akteur*innen, Positionen und Prozesse auf unterschiedlichsten Ebenen gelagert sein - etwa sensibilisiert Oberle (2012) für die Wahrnehmung der EU als «Mehrebenensystem». Und auch für den Politikunterricht in der Schweiz scheint eine Auseinandersetzung mit dem Ineinander von Ebenen und der zugehörigen Vielfalt von Beteiligten und Interessen von Relevanz - im Sinne eines "Doing European» (Richter, 2004) einschliesslich derjenigen der Lernenden. Im Folgenden interessieren wir uns anknüpfend an diese Überlegungen dafür, inwieweit im beobachteten Unterricht Interessen von Akteur*innen und deren Vielfalt sowie damit verbundene konfliktive Aushandlungsprozesse in den Blick geraten und dem Prinzip der Kontroversität Rechnung getragen wird (vgl. für eine Einzelfallanalyse mit demselben Anliegen bereits Thyroff und Waldis, 2020).

\section{Empirischer politikdidaktischer Forschungsstand}

Erkenntnisse dazu, welche Dimensionen des vielschichtigen Gegenstands «Europa» tatsächlich im Politikunterricht vorkommen, gibt es kaum. Die politikdidaktische Forschung beschränkt sich im deutschsprachigen Raum bislang darauf, Wissen, Konzepte, Kompetenzen oder Einstellungen bei Schüler*innen in Bezug auf Europa bzw. die EU zu erheben (z. B. Manzel, 2007; Oberle, 2012; Oberle \& Forstmann, 2015a; Weisseno \& Eck, 2009).

Befunde zu Wissen und Einstellungen bei Schüler*innen in der Schweiz liegen durch Stamm (2007) sowie die TEESAEC-Studie (z.B. Ziegler \& Reinhardt, 2012) vor. Im Rahmen zweitgenannter trat zutage, dass Lernende in der Schweiz über ein im Vergleich zu denjenigen in Deutschland unterdurchschnittliches Wissen zur EU verfügen (Reinhardt, Waldis, \& Ziegler, 2010, S. 191) und die EU für «ein komplexes, abstraktes und für die eigene Lebenswelt bedeutungsloses Gebilde» halten, wobei sie das Desinteresse mit der Nichtbeteiligung der Schweiz begründen (Wyss \& Schneider, 2012, S. 69). Interesse an Europa äussern die Lernenden primär auf touristischer Ebene, ein Befund, der an eine Studie Stamms (2007) anschliesst, die ein primär utilitaristisches, am eigenen Nutzen orientiertes Interesse an Europa konstatiert.

Ergebnisse zu Einstellungen und Vorstellungen von Schweizer Lehrpersonen liegen durch Aschwanden (2012) vor. Sie folgert, dass eine Mehrheit der Lehrpersonen an der EU interessiert ist - dazu auch Wyss und Schneider (2012, S. 64) - und dass eine Mehrheit «[e]ine Betroffenheit der Schweiz durch die EU» wahrnimmt (Aschwanden 2012, S. 78, 85). Gleichzeitig herrsche aber auch eine «Europaskepsis in der Schweizer Lehrerschaft» vor, die die Autorin 
auf die "Komplexität des Themas» und «die diffuse Erscheinung der EU und ihrer Handlungen» zurückführt (S. 83-84). Wichtig sei den Lehrpersonen die Vermittlung eines "Allgemeinwissen[s]», zu dem sie die Einigungsgeschichte, organisatorische Aspekte, Vor- und Nachteile einer Mitgliedschaft und die Positionierung der Schweiz zählen, wohingegen ein «Doing European" nach Richter (2004) und ein Blick auf das Mehrebenensystem der EU abwesend seien, ebenso wie anzubahnende Kompetenzen (Aschwanden, 2012). Aschwanden folgert, dass Lehrpersonen die «Dynamik und Mannigfaltigkeit des Themas Europa noch nicht für den Schulalltag erkannt» hätten (S . 86). Dass Lehrpersonen die Komplexität der EU Schwierigkeiten bereitet und die Ausbildung von Lehrpersonen in diesem Feld «defizitär» ist, geht auch aus einer Studie mit deutschen Lehrpersonen hervor (Oberle \& Forstmann, 2015b, S. 193-207).

Vorliegende Befunde zur Perspektive von Lehrpersonen und die noch schwache institutionelle Verankerung Politischer Bildung in der Schweiz legen also insgesamt die Vermutung nahe, dass die unterrichtliche Umsetzung des Gegenstands EU/Europa und die Realisierung einer genuin politischen Perspektive für Lehrpersonen herausfordernd sein dürften. Um dem jedoch genauer nachzuspüren, sind über die Erhebung von Einstellungen und Vorstelllungen hinaus auch Analysen von Unterrichtsprozessen notwendig, die zum Thema EU/Europa in der Politikdidaktik bislang kaum vorliegen und erstmals im Rahmen des dem Artikel zugrundeliegenden Forschungsprojekts durchgeführt werden (Thyroff \& Waldis, 2020; Waldis et al. 2017; weitere in Vorbereitung).

\section{Politische Fachlichkeit auf Kompetenzebene: Theoretische \& empirische Ausgangslage}

Wir schildern nun die relevanten theoretischen und empirischen Bezüge im Hinblick auf den zweiten Gegenstand unserer Analyse, die im Unterricht mittels Arbeitsaufträgen adressierten politischen Kompetenzen.

\section{Theoretische überlegungen}

Ähnlich wie in anderen Fachdidaktiken entstanden für Politische Bildung mehrere Entwürfe für im Unterricht zu fördernde Kompetenzen und bis heute gibt es keinen (inter-)nationalen Konsens über essentielle Kompetenzen politischer Bildung (Kennedy, 2008). Dennoch fällt bei genauerer Analyse der einzelnen theoretischen Vorstellungen auf, dass sie sich bei aller Unterschiedlichkeit in Terminologie und Zielsetzung oft auf gemeinsame Facetten beziehen. In nahezu sämtlichen Kompetenzmodellen und/oder Bildungsstandards werden die Ebenen Erkenntnisgewinnung, Urteilsbildung, Handlung sowie konzeptionelles Wissen aufgeführt (z.B. GPJE, 2004; Krammer, 2008; international z. B. 
Zhang, Tourney-Purta, \& Barber, 2012).

Als theoretische Grundlage für die Analyse beziehen wir uns auf das Modell von Krammer (2008), weil es am gründlichsten in Teiloperationen zerlegt wurde und damit am transparentesten offenlegt, wie die einzelnen Kompetenzbereiche verstanden werden. Krammer unterscheidet die Kompetenzbereiche Sach-, Methoden-, Urteils- und Handlungskompetenz. Sachkompetenz, bei der es um die reflektierte Auseinandersetzung mit fachlichen Begriffen, Kategorien und Konzepten geht, wird ihrerseits von deklarativen Wissensbeständen abgegrenzt, welche als Arbeitswissen bezeichnet werden. Die Methodenkompetenz wird in zwei Subdimensionen unterteilt: die Analyse bestehender und Erstellungen neuer Manifestationen. Ähnlich wird die Urteilskompetenz operationalisiert: in die Analyse bestehender und Entwicklung eigener Urteile. Zur Handlungskompetenz zählen zwei recht verschiedene Dimensionen: die Artikulation und Durchsetzung von Interessen sowie die Nutzung verschiedener Angebote von politischen Institutionen (Krammer, 2008).

\section{Empirischer politikdidaktischer Forschungsstand}

In der Politikdidaktik ist bisher kaum Grundlagenforschung hinsichtlich Aufgabenkultur und Kompetenzorientierung im Unterricht betrieben worden. Zwar existieren einige Studien mit dem Anspruch, Konzeptaufbau zu erforschen, allerdings wurde dabei eher deklaratives Wissen statt politischer Sachkompetenz gemessen (Hahn-Laudenberg, Weisseno, \& Eck, 2010; Reinhardt, Waldis, \& Ziegler, 2010). Von Manzel (2007) liegt eine quasi-experimentelle Studie vor, in der sie den Einfluss des Lehr-Lern-Settings auf die Urteils- und Analysefähigkeit untersucht. Dabei konnten durch Web Quests höhere Kompetenzzuwächse als durch Frontalunterricht erzielt werden. Gronostay (2016) untersuchte die Urteilskompetenz von Lernenden.

Zur Realisierung von Kompetenzorientierung im Unterricht liegt lediglich eine Studie von Manzel und Sowinski (2014) vor, nämlich zur Frage, welche kognitiven Prozesse durch Lernaufgaben angeregt wurden. Sie zeigen, dass reproduktive Aufgaben überwiegen, was zugleich bedeutet, dass kompetenzorientierte, d. h. selbstständige Problemlösung einfordernde Unterrichtssettings kaum vorkamen.

Relevant in unserem Zusammenhang sind weiter Studien zu Kompetenzorientierung im Geschichtsunterricht, weil es sich bei den von uns beforschten Lehrpersonen um Geschichtslehrpersonen handelt. Sowohl Studien in der Schweiz (Waldis, Hodel, \& Fink, 2012) als auch in Deutschland (Spiess, 2014) kamen zu dem Befund, dass ein «stofforientierter Zugang (...) mit Fokus auf dem Wissenserwerb» vorherrscht (Waldis et al., 2012, S. 154).

Aus diesen Forschungsergebnissen heraus lässt sich vermuten, dass auch im gegenwärtigen Politikunterricht wenige Unterrichtssettings vorkommen, die kompetenzorientiert angelegt sind. 


\section{Stichprobe, Datenerhebung und Datenanalyse}

Die untersuchten Unterrichtsstunden entstanden im Rahmen des eingangs vorgestellten Projekts. Darin nahmen Geschichtslehrpersonen zunächst an einer eineinhalbtägigen Weiterbildung teil ${ }^{3}$, in der fachliche Prinzipien der Politischen Bildung, z.B. das Kontroversitätsgebot, politische Basiskonzepte nach Sander (2009) und politische Kompetenzen vorgestellt wurden. Weiter wurde in die Lehrplanthemen "Europa-Schweiz» und «Menschenrechte» eingeführt und die je verschiedenen Charakteristika von Politischer Bildung und Geschichte gegenübergestellt. Eine Übersetzung in konkrete Unterrichtsarrangements erfolgte in dieser Phase noch nicht, sondern wurde den Lehrpersonen überlassen, die im Anschluss an die Weiterbildung je eine Unterrichtseinheit zu einem der beiden Lehrplanthemen unterrichteten. Diese Erprobung wurde durch ein fachspezifisch-pädagogisches Coaching (Staub, 2001) begleitet. Dieser Typus des Coachings sieht einen Austausch über vorab durch die Lehrperson erstellte Lektionsskizzen vor, wobei die Lehrperson die Entscheidungshoheit behält und instruktionale Elemente seitens der Coaches möglichst zu vermeiden sind. Die Funktion der Coaches besteht somit darin, die Lehrpersonen durch fachdidaktische Fragen in ihrer Reflexion über die Lektionsskizze zu begleiten.

Für die jetzige Analyse wurden die acht Doppellektionen (je 90 min) derjenigen vier Lehrpersonen herangezogen, die sich für das Thema "Europa Schweiz» entschieden haben. Wie in der Einleitung angekündigt, interessiert uns in diesem Artikel, wie die Lehrpersonen ihren Unterricht angebotsseitig gestalten. Das Zusammenwirken von Weiterbildung, Coachinggesprächen und Unterricht wird erst in künftigen Analyseschritten unser Fokus sein.

Der Unterricht wurde mit standardisiertem Kameraskript in Anlehnung an Hodel und Waldis (2007) videografiert und transkribiert. Nähere Angaben zur Stichprobe sind in Tabelle 1 aufgeführt.

Tabelle 1: Soziodemographische Daten der Lehrpersonen

\begin{tabular}{l|c|c|c|c}
\hline Lehrperson (LP) & LP4 & LP7 & LP10 & LP11 \\
\hline Alter & 44 & 39 & 28 & 53 \\
\hline Geschlecht (m/w) & $\mathrm{m}$ & $\mathrm{m}$ & $\mathrm{w}$ & $\mathrm{m}$ \\
\hline $\begin{array}{l}\text { Berufserfahrung als } \\
\text { Geschichtslehrperson }\end{array}$ & 13 & 11 & 2 & 3 \\
\hline $\begin{array}{l}\text { Schultyp/ } \\
\text { Schulstufe }\end{array}$ & $\begin{array}{c}\text { hohe } \\
\text { Anforderungen } \\
\text { Kanton }\end{array}$ & $\begin{array}{c}\text { Anforderungen } \\
9\end{array}$ & $\begin{array}{c}\text { hohe } \\
\text { Anforderungen } \\
8\end{array}$ & $\begin{array}{c}\text { Anforderungen } \\
9\end{array}$ \\
\hline AG & AG & AG & SO \\
\hline AG & & & & \\
\hline
\end{tabular}

Anmerkung: Die Kantone verfügen je über ein dreigliedriges Schulsystem: Basale, erweiterte und hohe Anforderungen. 
Die Lektionen wurden mittels qualitativer Inhaltsanalyse nach Kuckartz (2014) programmgestützt mit MAXQDA analysiert. In beiden Analysebereichen fokussieren wir nachfolgend auf Äusserungen der Lehrpersonen, also darauf, wie sie den Unterricht angebotsseitig gestalten.

Für die Betrachtung der Gegenstandesebene (Forschungsfrage 1) suchen wir solche Materialstellen, die sich als Behandlung der politischen Dimension im engeren Sinn kategorisieren lassen (vgl. hierzu obige theoretische Ausführungen in Abschnitt 2.1 sowie Vorarbeiten in Thyroff, Hedinger und Waldis, 2019) ${ }^{4}$, also in denen Akteure und deren (gegenläufige) Interessen sowie Aushandlungsprozesse thematisiert werden. Dabei beschränken wir uns auf zwei Unterrichtsphasen, die bei allen vier Lehrpersonen vorkommen und zumindest potenziell geeignet sind, eine solche politische Dimension im engeren Sinn sichtbar zu machen: die Thematisierungen 1) des europäischen Integrationsprozesses sowie 2) des Verhältnisses zwischen Schweiz und Europa. Wir erschliessen, inwieweit eine politische Dimension im engeren Sinne darin tatsächlich vorkommt, und nehmen eine interpretierende Gesamtschau über alle Lehrpersonen vor.

Hinsichtlich der adressierten Kompetenzen (Forschungsfrage 2) erfolgte die Auswertung deduktiv anhand des Kompetenzmodells von Krammer (2008). Hierfür wurden die Kompetenzbereiche und deren zugeordnete Fähigkeiten in ein Kategoriensystem überführt. Petrik (2010) kritisierte an Krammers Modell berechtigterweise die zahlreichen Überschneidungsbereiche (S. 145), sodass das Kompetenzmodell für die empirische Analyse insofern angepasst wurde, dass überlappende Teilkompetenzen lediglich einer Kernkompetenz zugewiesen wurden. Während mehrerer Kodierrunden wurde das Kategoriensystem geschärft und so weiterentwickelt, dass jede im Unterricht erteilte Aufgabenstellung einer Kompetenz zugeordnet werden konnte ${ }^{4}$. Gemäss der Fragestellung wurden die Aufgabenstellungen der Lehrpersonen den Kategorien zugeordnet. Als Aufgabenstellungen wurden hier alle Arbeitsanweisungen verstanden, die die Lehrpersonen den Lernenden erteilten, sei es in mündlicher oder schriftlicher Form.

\section{Ergebnisse}

\section{Zum Politischen auf Gegenstandsebene 5}

Nachfolgend beschreiben wir zunächst, inwiefern auf Gegenstandsebene das Politische, also Akteurs- und Interessenvielfalt und damit verbundene Aushandlungsprozesse, in den Blick geraten und Kontroversität realisiert wird.

Thematisierung des europäischen Integrationsprozesses Alle Lehrpersonen verorten den Ausgangspunkt für eine europäische Zusammenarbeit in der Zeit nach dem Zweiten Weltkrieg und führen als Motiv Frieden an, womit sie sich in Übereinstimmung dazu bewegen, wie auch in wissenschaftlichen Darstellungen der Ursprung der europäischen Zusammenarbeit gedeutet 
wird (Jaag, 2009). Einige Lehrpersonen gehen zudem darauf ein, dass sich mit der Friedensidee auch eine wirtschaftliche Zusammenarbeit bzw. der Wunsch nach Wohlstand kombiniert habe. Der in der Montanunion verwirklichte gemeinsame Markt für Kohle und Stahl wird von LP7 eher abstrakt als «Mittel» zum "Zweck» zur Friedenssicherung behandelt (LP7, Doppellektion (DL) 1), LP10 konkretisiert als einzige Lehrperson, es sei dabei darum gegangen, «dass da kein Land irgendwie aufrüsten kann» (LP10, DL1).

Im weiteren Verlauf der Auseinandersetzung mit dem europäischen Integrationsprozess geraten konkrete Motive von beteiligten Akteur*innen dann hingegen kaum noch in den Blick. Dominierender Modus, welcher sich bei drei Lehrpersonen (LP7, LP10, LP11) zeigt, ist das Darstellen des Integrationsprozesses als lineares und quasi selbstläufiges Aufeinanderfolgen von Bündnissen, Verträgen und hinzukommenden Mitgliedsstaaten:

LP7: [...] Ehm und eh diese Zusammenarbeit hat sich dann weiterentwickelt/ weiterentwickelt das gab dann die Römischen Verträge, da habt ihr gelesen, dass man vor allem in Wirtschaftsfragen und Nuklearfragen zusammengearbeitet hat. Dann kam dieses Schengener Abkommen, das ist heute noch ein Begriff, da ist ehm eh das freie Reisen ein Thema, also keine Personenkontrollen zunächst beschränkt auf einige wenige Staaten [...]. (LP7, DL2)

Es zeigt sich ein entlang der Chronologie orientiertes, ereignisgeschichtliches Erzählen, zu dem die Lehrpersonen mutmasslich aufgrund ihres Hintergrunds als Geschichtslehrpersonen neigen und das einzelne von ihnen mit dem Mittel des Zeitstrahls visualisieren (LP7, LP10) sowie mithilfe aneinandergereihter Karten (LP7, LP10, LP11), wodurch die europäische Integration zugleich als Prozess der zunehmenden geographischen Ausdehnung erscheint. Einzig bei LP10 steht nicht die geographische Ausdehnung, sondern vielmehr die Vertiefung einer inhaltlichen Zusammenarbeit im Zentrum des Zeitverlaufs:

LP10: Jetzt haben wir kurz die Entwicklungsgeschichte der EU angeschaut, wir haben gesehen, es ist eine zunehmend verstärkte Zusammenarbeit, zuerst wirtschaftlich, anschliessend dann auch zunehmend auch eine politische Zusammenarbeit. (LP10, DL1)

Motive im engeren Sinn, die eine Verstärkung der europäischen Integration begründen könnten, geraten jedoch kaum in den Blick. Lediglich LP10 präzisiert:

LP10: So (..) in dieser Zeit trete immer mehr Länder, hm, dieser Europäischen Gemeinschaft bei. Könnt ihr euch vorstellen, warum ein Land beschliesst, (.) dieser Gemeinschaft beizutreten? [...]

[..]

LP10: Mhm (bejahend). Also es ist eine Erleichter-/ es geht / sind wirtschaftliche Vorteile, die ein Land hat, wenn es der EU beitritt, hm, genau, hauptsächlich. (LP10, DL1) 
Ebenfalls einzig LP10 geht darauf ein, dass der für die Zusammenarbeit initiale Wunsch nach «Wohlstand und Frieden» auch für die spätere Institution EU leitend sei. Differenziertere Interessen, etwa abhängig vom einzelnen Nationalstaat, geraten nicht in den Blick. Zwar werden von einem Teil der Lehrpersonen durchaus historische Kontexte thematisiert, die die Ausgangslage für das Dazukommen weiterer Mitgliedsstaaten bilden, etwa die Auflösung von Diktaturen. Welches die konkreten Motive einzelner Staaten gewesen sein könnten, um Mitglied europäischer Organisationen zu werden, bleibt gleichwohl unklar, vielmehr wird bei zwei Lehrpersonen (LP7, LP11) der Eindruck eines quasi zwangsläufigen Wegs erweckt, den Staaten von der Diktatur hin zur Demokratie und schliesslich EU-Mitgliedschaft durchlaufen:

LP7:[...] Aber die Logik ist die, und zwar diese Diktatur / sowohl in / eh wo sind wir da / in Spanien als auch in Portugal die sind so in Mitte 70er Jahren / 1974, 75 werden die abgeschafft und dann braucht das Land gewisse Zeit um sich zu erholen, diese Werte von Demokratie und Friede und so ehm eh eeew pff einzuführen sozusagen / und dann treten die beiden Staaten ehm der EU bei. Also das ist der Zusammenhang. Ok? Wir haben Diktaturen in verschiedenen Staaten, sei das Portugal oder Spanien und ich nehme vorweg, übrigens auch in Griechenland, das ist ne ähnliche Geschichte. Auch Griechenland hat ne Diktatur und die müssen sich so demokratisieren und dann treten sie der EU bei. (LP7, DL1)

Das von den Lehrpersonen thematisierte Akteursspektrum ist begrenzt: In der Regel sind es diverse einzelne Nationalstaaten oder Organisationen in ihrer Gesamtheit (z. B. Montanunion, EU), die als Ganzes handeln, ausserdem kommen akteursverschleiernde Formulierungen wie «man» und «sie» oder vereinzelt Einzelpersonen (Churchill, Marshall) vor. Eine weitere Ausdifferenzierung des Akteursspektrums, z.B. innerhalb der Ebene von Nationalstaaten, findet nicht statt.

Aushandlungsprozesse im Zusammenhang mit dem europäischen Integrationsprozess kommen praktisch nicht zum Tragen. Lediglich zwei Lehrpersonen (LP7, LP10) beschreiben den Prozess in abstrakter Weise als langwierig. Das Nebeneinander von unterschiedlichen, gegenläufigen Positionen kommt überhaupt nur bei LP7 zum Vorschein und dort nur in angedeuteter Form, ohne Positionen genauer zu bestimmen:

LP7: jetzt ihr könnt euch vorstellen, wenn sich da so 28 Staaten ehm zusammentun, oder auch nur sechs zu Beginn, dann gibt es einiges zu besprechen. Und einige Abmachungen ehm zu definieren. Und das war eine ziemliche Herausforderung. So viele Staaten irgendwie an einen Tisch zu bringen, für Frieden zu sorgen. Zusammenzuarbeiten, da gibt's verschiedene Sprachen, verschiedene Mentalitäten, verschiedene Interessen, ein Staat muss ja schlussendlich auch ein bisschen auf/ was heisst ein bisschen / ziemlich auf seine Interessen achten eh und deshalb war es nicht ganz einfach, ein Gebilde zu 
finden [...], die irgendwie für alle lebbar eh war. Und entsprechend dauerte das einen Moment, bis man eine mh ja eine Möglichkeit fand. (LP7, DL1)

In der Zusammenschau lässt sich schlussfolgern, dass in denjenigen Sequenzen, die den europäischen Integrationsprozess behandeln und potenziell eine Realisierung der politischen Perspektive und des Prinzips der Kontroversität erlauben würden, diese nur in begrenzter Weise zum Tragen kommt.

\section{Thematisierung des Verhältnisses Schweiz-Europa}

Deutlicher erscheinen diese Aspekte im Zusammenhang mit dem Verhältnis zwischen der Schweiz und Europa. Alle Lehrpersonen machen sichtbar, dass das Verhältnis in der Schweiz ein umstrittenes sei, dass es unterschiedliche Beteiligte und Positionen gebe - ein Themenbereich, der Lehrpersonen wichtig ist, wie bereits Aschwanden (2012) feststellte. Etwa behandeln alle Lehrpersonen erfolgte Abstimmungen (EWR-Beitritt, Masseneinwanderungsinitiative, Begrenzungsinitiative):

LP7: also das ist eine eher knappe Geschichte und anhand des Wahlresultates seht ihr auch, dass die ganze Sache sehr emotional und eng war, wenn wir solch enge Wahlresultate haben, kann man davon ausgehen, dass die Emotionen ziemlich im Spiel waren, weil da wurde hart debattiert, es wurde hart diskutiert und am Schluss hat die Schweiz das abgelehnt. [...]

Der Bundesrat stand eigentlich dafür ein, dass man dem EWR beitritt, weil das gewisse Vorteile ehm gehabt hätte. Das Schweizer Volk sagte aber ehm nein, es sagte nein genau bzw. wollte nicht zu dem EWR beitreten [...]

Die Deutschschweiz war eh ziemlich klarer eh dagegen, dass man zu dem EWR beitritt und die französische Schweiz hätte eigentlich dazugehören wollen. (LP7, DL2)

Stets sind es die Lehrpersonen oder die von ihnen eingesetzten Materialien, die über das Vorhandensein und Inhalte unterschiedlicher Positionen aufklären. Dies gilt auch für eine Pro-Contra-Debatte, die bei LP7 stattfindet und in der auf Basis vorgegebener Materialien zur Frage der eigenständigen Begrenzung von Zuwanderung durch die Schweiz argumentiert werden soll. Auch wenn es «fremde» Positionen sind und für die Debatte nur ein begrenztes Positionsspektrum zur Verfügung steht - für die Befürworterseite wird auf das Argumentarium des Initiativkommitees zurückgegriffen, das Material für die Gegnerseite basiert auf einem Positionspapier der FDP - wird hier in begrenztem und didaktisch reduziertem Masse Kontroversität realisiert (vgl. zu diesem Fall detaillierter Thyroff \& Waldis 2020).

Während in der genannten Debatte die eigenen Sichtweisen der Lernenden nicht im Zentrum stehen, kommt die persönliche Relevanz Europas an anderen Stellen durchaus zum Tragen. Etwa sollen die Lernenden bei derselben Lehrperson (LP7) auch überlegen, was sich für sie persönlich ändern würde, wenn es die Bilateralen Verträge nicht gäbe, wobei Aspekte wie durch die Verträge ermöglichtes erleichtertes bzw. vergünstigtes Reisen und Einkaufen in 
den Blick geraten, also stark wirtschaftlich bzw. utilitaristisch argumentiert wird - anknüpfend an die oben geschilderten Befunde von Stamm (2007).

Die Inhalte und Vor- und Nachteile der Bilateralen Verträge oder einzelner Teilbereiche wie Schengen geraten - in unterschiedlichem Ausmass - bei allen Lehrpersonen ins Blickfeld, teilweise abstrakt für die Schweiz, teilweise im Hinblick auf den Alltag der Lernenden. Konkrete Positionen der Lernenden in Bezug auf Europa und ihre Handlungsspielräume im Hinblick auf die Gestaltung von und Partizipation an Europa werden abgesehen davon jedoch nicht thematisiert oder reflektiert - einzig in Ansätzen im Fall von LP4, bei welcher die Auseinandersetzung mit dem Europarat, der Mitgliedschaft der Schweiz und dem Europäischen Gerichtshof für Menschenrechte im Zentrum steht und anhand einiger Beispielfälle sichtbar wird, dass auch Schweizer*innen und die Lernenden persönlich dort klageberechtigt wären (LP4, DL2).

\section{Zum Politischen auf Kompetenzebene}

Nachfolgend wenden wir uns der Ebene der adressierten Kompetenzen zu und der Frage, inwiefern im beobachteten Unterricht genuin politische Kompetenzen angebahnt werden. ${ }^{6}$ Wir klären dies wiederum deskriptiv entlang exemplarischer Materialauszüge, nun erweitert um quantitative Befunde. Obgleich Auszählungen angesichts der kleinen Stichprobe nur begrenzte Aussagekraft haben, erlauben sie doch einen Einblick hinsichtlich Schwerpunktsetzungen der Lehrpersonen.

\section{Tabelle 2: Häufigkeit der in den Aufgaben adressierten Fähigkeiten der Sachkompetenz}

\begin{tabular}{l|l|c}
\hline Kategorie & Ankerbeispiel & $N_{\text {Arbeitsaufträge }}$ \\
\hline Vorwissen aktivieren & $\begin{array}{l}\text { «Ich möchte, dass ihr euch zu Beginn zu zweit kurz } \\
\text { Gedanken macht, was aus eurer Sicht Europa ist.» }\end{array}$ & 11 \\
\hline Vorwissen vergleichen & $\begin{array}{l}\text { «Setzt euch in Gruppen zusammen und überlegt, } \\
\text { welche Sicht, welche Brille hattet ihr an, welche } \\
\text { Brille hattet ihr an? Was habt ihr drauf gehabt, was } \\
\text { die anderen nicht drauf gehabt haben?» }\end{array}$ & 2 \\
\hline $\begin{array}{l}\text { Präkonzepte mit erarbeiteten } \\
\text { Sachverhalten/Kategorien in } \\
\text { Beziehung setzen }\end{array}$ & $\begin{array}{l}\text { «Ihr habt Ende der ersten Lektion aufgeschrieben, } \\
\text { was ist für mich Europa? Eventuell könnt ihr } \\
\text { nach dem Interview euren eigenen Notizen, eurer } \\
\text { Reflexion, noch etwas hinzufügen.» }\end{array}$ & 4 \\
\hline
\end{tabular}

Im Bereich der Sachkompetenz (vgl. Tabelle 2) zeigt sich, dass den Lehrpersonen die Relevanz des Vorwissens der Lernenden für den Lehr-Lernprozess bewusst ist. Im Sinne konzeptuellen Lernens fordern drei Lehrpersonen (LP4, LP7, LP11) die Lernenden zu Lektionsbeginn auf, ihr Vorwissen zu verbalisieren. Allerdings wird während und nach den Erarbeitungsphasen nur von LP4 noch darauf zurückgegriffen, indem die Lehrperson die Lernenden während der Lektionen dazu auffordert, ihr erarbeitetes Wissen mit dem zu Beginn notierten Vorwissen 
abzugleichen. Da das Vorwissen der Lernenden somit nur vereinzelt verglichen und systematisiert oder überhaupt notiert wird, scheint es den Lehrpersonen eher um eine Aktivierung aller Lernenden anstatt der Anbahnung eines Conceptual Change zu gehen. ${ }^{7}$

Folgendes Beispiel soll das Zusammentragen der Lernendenvorstellungen (Phase 1 und 2) und den dann unmittelbar folgenden abrupten Übergang zum nächsten Unterrichtsgegenstand (Phase 3) illustrieren:

Phase 1: Erteilung Arbeitsauftrag (ab Minute 2:16)

LP7: «Ich möchte, dass ihr euch zu Beginn zu zweit kurz Gedanken macht, was aus eurer Sicht Europa ist.» [...]

Phase 2: Systematisierung Antworten durch Lehrperson (ab Minute 7:15) LP 7: «So, wenn wir schnell den Überblick uns verschaffen, dann finden wir mehr/ eh Mehrfachnennungen. Wir haben hier eine politische Komponente (räuspern) mit der EU oder halt einem Nationenbund. Es gibt auch eh so eher geografische (...)»[...]

\section{Phase 3: Textanalyse EGKS-Vertrag, Erteilung Arbeitsauftrag (ab Minute} 10:29)

LP 7: «(...) nämlich was ist der Inhalt dieses Textes, dann was ist der historische Kontext, also wenn wir die Jahreszahl anschauen, wie müssen wir diese diese Bestimmung oder diesen Vertrag einordnen und die dritte Frage ist, gibt's eine Strategie, die da eh die da umgesetzt werden soll?» (LP7, Auszug DL1)

Die Analysen im Bereich der Methodenkompetenz (vgl. Tabelle 3) offenbaren, dass Medien mit politischem Inhalt sehr einseitig dahingehend analysiert werden, welche Sachaussagen sich diesen entnehmen lassen. Dies verdeutlicht eine vorherrschende Stoff- statt Kompetenzorientierung. Nur ansatzweise werden weitergehende politische Fähigkeiten, wie die Analyse der zugrundeliegenden Intentionen oder auch die Auswirkungen politischer Entscheide, adressiert. Die für kritisches und analytisches Denken wichtige Denkoperation der Prüfung von Sachaussagen hinsichtlich ihrer sachlichen oder argumentativen Plausibilität wird nicht adressiert, ebenso wenig wie komplexere Fähigkeiten zur Analyse von Manifestationen des Politischen angebahnt werden. Lediglich von LP11 wurde zweimal das Vorgehen zur Lösung bestimmter Lernaufgaben thematisiert, ansonsten beschränkte es sich auf kurze Klärungen unbekannter Begriffe. Bei Betrachtung der einzelnen Lektionen dominiert zudem ein Einsatz vieler verschiedener Medien, denen lediglich Sachaussagen entnommen werden sollen, bevor dann das nächste Medium zum selben Analysezweck eingeführt wird. 
Tabelle 3: Häufigkeit der in den Aufgaben adressierten Fähigkeiten der Methodenkompetenz

\begin{tabular}{|c|c|c|}
\hline Kategorie & Ankerbeispiel & $N_{\text {Arbeitsaufträge }}$ \\
\hline Entnahme von Sachaussagen & $\begin{array}{l}\text { «Streicht doch an, worüber spricht Churchill in } \\
\text { seinem Text?» }\end{array}$ & 30 \\
\hline $\begin{array}{l}\text { Vergleich von Sachaussagen } \\
\text { aus mehreren Medien }\end{array}$ & $\begin{array}{l}\text { "Dazu habe ich euch da ein Blatt ausgeteilt, wo das } \\
\text { ganz kurz dargestellt wird. Vielleicht vergleicht ihr } \\
\text { diese Positionen auch mit dem, was ihr in dem Video } \\
\text { gesehen habt.» }\end{array}$ & 2 \\
\hline $\begin{array}{l}\text { Kontextualisierung von } \\
\text { Sachaussagen }\end{array}$ & $\begin{array}{l}\text { "Betrachte die Jahreszahlen der Beitritte [in die EU]. } \\
\text { Kannst du die Beitrittsjahre der Länder historisch } \\
\text { begründen?» }\end{array}$ & 6 \\
\hline $\begin{array}{l}\text { Benennen unbekannter } \\
\text { Begriffe }\end{array}$ & $\begin{array}{l}\text { "Jetzt möchte ich dass ihr den Text lest. Je nach dem } \\
\text { Wörter, die ihr nicht versteht (..) euch zu merken.» }\end{array}$ & 6 \\
\hline $\begin{array}{l}\text { Erkenntniswert von Medien } \\
\text { bewerten }\end{array}$ & $\begin{array}{l}\text { "Was sind Vor- und Nachteile Zeitzeugenarbeit im } \\
\text { Vergleich zum Buch?» }\end{array}$ & 1 \\
\hline $\begin{array}{l}\text { Qualität der Sachaussagen } \\
\text { prüfen }\end{array}$ & & 0 \\
\hline $\begin{array}{l}\text { Wirkung von Medien } \\
\text { analysieren }\end{array}$ & $\begin{array}{l}\text { «Denkt über die Wirkung der Rede nach. Churchills } \\
\text { Rede hatte eine grosse Wirkung. Sie wurde sehr } \\
\text { berühmt. Stellt Vermutungen an, weshalb die Rede } \\
\text { dieses Echo hatte, und notiert diese.» }\end{array}$ & 1 \\
\hline $\begin{array}{l}\text { Interessen/Intentionen } \\
\text { erschliessen }\end{array}$ & $\begin{array}{l}\text { "So (..) in dieser Zeit trete immer mehr Länder, } \\
\text { hm, dieser Europäischen Gemeinschaft bei. Könnt } \\
\text { ihr euch vorstellen, warum ein Land beschliesst, (.) } \\
\text { dieser Gemeinschaft beizutreten?» }\end{array}$ & 4 \\
\hline $\begin{array}{l}\text { Auswirkungen politischer } \\
\text { Entscheide analysieren }\end{array}$ & $\begin{array}{l}\text { "Vermute, welche Bedeutung die Personenfreizügi- } \\
\text { gkeit für deinen Alltag in Zukunft haben könnte.» }\end{array}$ & 3 \\
\hline $\begin{array}{l}\text { Vergleich Sachaussagen mit } \\
\text { öffentlicher Rezeption }\end{array}$ & & 0 \\
\hline $\begin{array}{l}\text { Recherchieren von Informa- } \\
\text { tionen }\end{array}$ & $\begin{array}{l}\text { "Ihr recherchiert über den Europäischen Gerichtshof } \\
\text { für Menschenrechte. Was tut der?» }\end{array}$ & 2 \\
\hline $\begin{array}{l}\text { Reflexion methodischen } \\
\text { Vorgehens }\end{array}$ & $\begin{array}{l}\text { "Während einige noch fertig schreiben, darf ich } \\
\text { schnell fragen, wie seid ihr vorgegangen?" }\end{array}$ & 2 \\
\hline $\begin{array}{l}\text { Durchführung eigener } \\
\text { Studien }\end{array}$ & & 0 \\
\hline
\end{tabular}

Das folgende Beispiel soll dies illustrieren. Es zeigt den wesentlichen Verlauf des ersten Teils einer Doppellektion anhand der darin formulierten Arbeitsaufträge:

Phase 1: Ansehen eines Erklärvideos über Bilaterale Verträge und Grundfreiheiten

LP 11: «Die Schweiz ist also nicht in der EU. Da würde ich mal dazu ein kleines Video so erklärend abspielen lassen.» [...]

LP 11: «Also in diesem Video wurde jetzt da viel gezeichnet und gesprochen, was ist euch so geblieben? Was fandet ihr das Wichtigste was ihr jetzt gesehen habt?» $[\ldots]$ 


\section{Phase 2: Austeilen eines Arbeitsblattes zu Bilateralen Verträgen und Grund- freiheiten}

LP 11: «Ich habe euch ein Arbeitsblatt gemacht, oben ist nochmal der Begriff bilateral erklärt. Die Bilateralen Verträge. Es geht darum also die Beziehung der Schweiz zur Europäischen Union da ein bisschen auseinander zu nehmen, die sind geregelt, so wie das im Video gesagt hat, mit bilateralen Verträgen (...) kannst du mal lesen S9?» [...]

LP 11: «Und dann bestehen auf diesem Binnenmarkt der EU vier Grundfreiheiten. Das ist freier Personenverkehr, freier Warenverkehr, freier Dienstleistungsverkehr und freier Kapitalverkehr. (...) jetzt tauscht euch mal aus, was da unter den einzelnen (...) zu verstehen ist.» [...]

\section{Phase 3: Austeilen eines Arbeitsblattes zu den Positionen Schweizer Parteien zur EU}

LP 11: "Jetzt will ich da mal anschauen, wie das aussieht jetzt in der Schweiz, wie die Parteien zu dieser EU stehen. Dazu habe ich euch da ein Blatt ausgeteilt, wo das ganz kurz dargestellt wird. Vielleicht vergleicht ihr diese Positionen auch mit dem, was ihr in dem Video gesehen habt.» (LP11, Auszug DL2)

Trotz schlanker Rahmenpläne mit wenigen verpflichtenden inhaltlichen Vorgaben scheinen die Lehrpersonen zu versuchen, in den zwei Doppellektionen möglichst viel inhaltlichen Input in die Stunden zu legen, wodurch für Denkleistungen seitens der Lernenden kaum Zeit bleibt und sie demzufolge lediglich dazu aufgefordert werden, Informationen aus Materialien zu entnehmen, die anschliessend nicht hinterfragt werden.

Aus diesen Erkenntnissen heraus erscheint es folgerichtig, dass für Aufgaben, die auf eine Förderung der Urteilskompetenz abzielen, kaum Zeit bleibt. Lediglich LP7 nutzt 40 Minuten der 180-minütigen Unterrichtseinheit für eine Pro-Contra-Debatte. Dies ist die einzige Sequenz, in der Aspekte von Urteils- und Handlungskompetenz gefördert werden. Ansonsten verzichten die Lehrpersonen darauf, ihre Lernenden zur individuellen wie kollektiv-diskursiven Urteilsbildung anzuregen.

\section{Synthese}

In der Zusammenschau über die beiden Analysebereiche zeigt sich, dass genuin politische Fachlichkeit in den Lektionen bedingt zu beobachten ist. Während auf Gegenstandsebene die Thematisierung des europäischen Integrationsprozesses weitgehend ohne die Nennung konkreter und divergierender Interessen und Aushandlungsprozesse auskommt, wird das Verhältnis aus Schweiz und Europa von allen Lehrpersonen als kontroverses dargestellt. Vereinzelt haben die Lernenden Gelegenheit, solche Positionen zu erarbeiten oder gar aus deren Perspektive heraus zu argumentieren, wobei es primär fremde und nicht die 
eigenen Perspektiven sind. Hierzu ergänzend erweisen sich die Ergebnisse des zweiten Analysestrangs, dass Urteils- und Handlungskompetenz nur in einem Fall angebahnt wird und dass Konzepte der Lernenden zwar zu Beginn der Lektionen erfragt werden, später aber selten nochmals relevant werden. Die Lernenden selbst als Teil des Politischen zu verstehen, so lässt sich zusammenfassend folgern, kommt insofern kaum vor, und es liegt in weiten Strecken ein an Wissensvermittlung orientierter Unterricht vor, etwa wenn beim Umgang mit Materialien die Entnahme von Sachaussagen zentriert wird oder Kontroversität durch Lehrperson und Materialien berichtet wird.

Abschliessend möchten wir auf zwei Limitationen unserer Studie eingehen und ausgehend davon weiteren Forschungs- und Handlungsbedarf bestimmen. Zunächst wird kein Anspruch erhoben, aus der kleinen untersuchten Stichprobe breite Rückschlüsse auf die Mehrheit der Lehrpersonen zu ziehen, die bereits jetzt oder im Zuge der Lehrplaneinführung in näherer Zukunft Politische Bildung unterrichten werden - sei es ohne oder mit Besuch einer Weiterbildung. In der mosaikartigen Zusammenschau mit weiteren vorliegenden Befunden zu Vorstellungen von Politischer Bildung (Allenspach, 2014; Da Rin \& Künzli 2006) und unterrichtlichen Umsetzungen (z. B. Bürgler \& Hodel, 2012; Thyroff, Hedinger, \& Waldis 2019) verdichtet sich jedoch derzeit ein Bild, demzufolge fachliche Prinzipien, Perspektiven und Kompetenzen der Politischen Bildung bislang im Unterricht zu wenig berücksichtigt sein dürften.

Da die von uns beforschten Lehrpersonen nur nach Besuch der Weiterbildung beim Unterrichten Politischer Bildung gefilmt werden, können wir zweitens nicht nachvollziehen, welchen (positiven oder negativen) Effekt die Weiterbildung und das begleitende Coaching auf deren Unterricht hatten. Eine vertiefte Analyse der von uns gefilmten Coachinggespräche im Hinblick auf diese Frage steht noch aus. Welche Weiterbildungsformate in der Politischen Bildung inwiefern (nicht) wirken, wurde von der Politikdidaktik bislang generell kaum erforscht, sodass sich diesbezüglich erheblicher Forschungsbedarf, vor allem im Rahmen kontrollierter Interventionsstudien, ergibt. Befunde aus Weiterbildungsstudien anderer Domänen deuten insgesamt darauf hin, dass vor allem langfristige Massnahmen, bei welchen Zeit zur «Verarbeitung der Fortbildungsinhalte» zur Verfügung steht und bei welchen eine «Verschränkung von Input-, Erprobungs- und Reflexionsphasen» stattfindet, erfolgversprechend sind (Lipowsky \& Rzejak, 2012, S. 7). Inhaltlich böten sich auf Basis unserer Unterrichtsbeobachtungen insbesondere solche Weiterbildungsformate und zugehörige Forschungen an, die Lehrpersonen dazu befähigen sollen, eine genuin politische Perspektive auf Themen einzunehmen, politisches Konzeptlernen zu intensivieren und gezielt politische Urteils- und Handlungskompetenz bei Schülerinnen und Schülern zu fördern.

Solche auf Langfristigkeit angelegten Weiterbildungsformate sind freilich zeitaufwändig und es ist fraglich, inwieweit Lehrpersonen diese neben dem regulären Tagesgeschäft bewältigen können. Die Politische Bildung steht also derzeit vor dem Spagat, dass sie zwar eine neue Chance durch flächendeckende 
Festschreibung im Lehrplan 21 erhält, dass es für ihre adäquate Umsetzung aber auch ausreichender Ressourcen bedürfte. Dies gilt nicht nur für die Weiterbildung, sondern auch für die Ausbildung angehender Lehrpersonen. Für diese erachten wir es als notwendig, dass Politische Bildung den Status als dritte, eigenständige fachliche Säule innerhalb des Faches RZG erhält, also ihr derselbe Stellenwert wie Geschichte und Geographie eingeräumt wird, damit die fachlichen und fachdidaktischen Prinzipien Politischer Bildung gleichberechtigt durchdrungen werden können.

\section{Anmerkungen}

1 Wir danken ausserdem Franziska Hedinger als weiteres Mitglied unseres Projektteams für ihre Mitarbeit bei der Erhebung der hier verwendeten Daten und für ihre Lektüre und Anregungen zu unserem Beitrag.

2 Die Ausführungen in Abschnitt 2 finden sich strukturähnlich bereits in einer früheren Projektpublikation (Thyroff \& Waldis, 2020).

3 Die Ausschreibung der Weiterbildung mit begleitender Forschung erfolgte im Kursprogramm der PH FHNW, Lehrpersonen meldeten sich freiwillig an. Es ist also davon auszugehen, dass es sich um Personen handelt, die für die Realisierung Politischer Bildung motiviert sind.

4 Die «politische Dimension im engeren Sinn» stellt für uns eine inhaltsanalytische Kategorie dar. Sie wurde im Rahmen einer früheren Analyse von Einstiegssequenzen zum Thema Menschenrechte theorie- und materialbasiert entwickelt und dort neben weiteren Dimensionen (rechtliche, moralische, historische) eingesetzt. (Thyroff, Hedinger \& Waldis, 2019, 97, 101-102). Mit dem vorliegenden Text bauen wir auf diesen Vorarbeiten auf, nun mit Fokus auf die politische Dimension im engeren Sinn und anhand von Lektionen zum Thema Europa. Eine Intercoderreliabilität wurde für diesen Analysestrang nicht berechnet, jedoch das Kategoriensystem im Rahmen der genannten früheren Analysen im interpersonellen Austausch diskutiert und geschärft.

5 Strittige Fälle wurden im Forscherteam besprochen. Die Reliabilität der Codierungen wurde im späteren Verlauf des Analyseprozesses an weiteren Lektionen errechnet, die das Thema Menschenrechte behandelten und nicht in die hier vorgelegte Analyse eingeflossen sind. Dort ergab sich eine moderate Interraterreliabilität zwischen zwei Kodierenden für das gesamte Kategoriensystem im Bereich Kompetenzen $(k=0.61)$ und für die vier einzelnen Kompetenzbereiche $(k=0.68)$. Vgl. dazu: Scheller \& Thyroff $(2020$, im Druck).

6 Zu einer der vier Lehrpersonen existiert bereits eine Einzelfallanalyse hinsichtlich der Frage, wie sich die politische Dimension auf Gegenstandsebene manifestiert (Thyroff \& Waldis, 2020). Einzelne dort bereits vorgestellte Befunde tauchen nun erneut auf, nun aber im Kontext weiterer analysierter Einheiten.

7 Eine Analyse aller Stunden des Gesamtprojekts zu den Themen «Europa - Schweiz» sowie «Menschenrechte» wird an anderer Stelle veröffentlicht (Scheller \& Thyroff, 2020, im Druck).

8 Eine detaillierte Analyse der Stunden hinsichtlich des konzeptuellen Lernens ist an anderer Stelle eingereicht (Scheller, Thyroff, Schneider, \& Waldis, eingereicht). 


\section{Literaturverzeichnis}

Allenspach, D. (2014). Verständnisse Deutschschweizer Lehrpersonen von politischer Bildung: Annahmen und Befunde. In B. Ziegler (Hrsg.), Vorstellungen, Konzepte und Kompetenzen von Lehrpersonen der Politischen Bildung. Beiträge zur Tagung «Politische Bildung empirisch 2012» (Politische Bildung in der Schweiz 3, S. 14-36). Zürich: Rüegger.

Aschwanden, G. (2012). Einstellungen und Vorstellungen von Lehrkräften zur Europabildung in der Schule. In B. Ziegler \& V. Reinhardt (Hrsg.), Was Schweizer Jugendliche von der EU wissen - Die schweizerische TEESAEC-Studie, herausgegeben (S. 75-98). Zürich: Rüegger.

Bürgler, B., \& Hodel, J. (2012). Die spolitische Perspektives im Unterricht - Erkenntnisse einer Videoanalyse von Geschichts- und Politikunterricht. In D. Allenspach \& B. Ziegler (Hrsg.), Forschungstrends in der politischen Bildung. Beiträge zur Tagung "Politische Bildung empirisch 2010» (S. 51-62). Zürich: Rüegger.

Da Rin, S., \& Künzli, S. (2006). Forschungsbericht zur explorativen Studie "Umsetzung von politischer Bildung in der Volksschule». Zürich: PHZH, Departement Forschung und Entwicklung.

Gesellschaft für Politikdidaktik und politische Jugend- und Erwachsenenbildung. (GPJE). (2004). Nationale Bildungsstandards für den Fachunterricht in der Politischen Bildung an Schulen. Entwurf. Schwalbach: Wochenschau.

Grammes, T. (2014). Kontroversität. In W. Sander (Hrsg.), Handbuch politische Bildung (4. Aufl., Politik und Bildung 69, S. 266-274). Schwalbach: Wochenschau.

Gronostay, D. (2016). Argument, Counterargument, and Integration? Patterns of Argument Reappraisal in Controversial Classroom Discussions. Journal of Social Science Education 15(2), 42-56.

Hahn-Laudenberg, K., Weisseno, G., \& Eck, V. (2010). Wie verknüpfen Schüler/-innen Fachkonzepte in Lernaufgaben? Eine computergestützte Analyse von Schülerantworten aus der TEESAEC-Studie. In I. Juchler (Hrsg.), Kompetenzen in der politischen Bildung, (S. 159-168). Schwalbach: Wochenschau.

Heldt, I. (2018). Die subjektive Dimension von Menschenrechten. Zu den Implikationen von Alltagsvorstellungen für die Politische Bildung (Bürgerbewusstsein. Schriften zur Politischen Kultur und Politischen Bildung). Wiesbaden: Springer VS.

Hodel, J., \& Waldis, M. (2007). Sichtstrukturen im Geschichtsunterricht - die Ergebnisse der Videoanalyse. In P. Gautschi, D. V. Moser, \& K. Reusser (Hrsg.), Geschichtsunterricht heute. Eine empirische Analyse ausgewählter Aspekte (S. 91-142). Bern: hep.

Jaag, T. (2009). Europarecht. Die europäischen Institutionen aus schweizerischer Sicht (2. Aufl.). Zürich: Schulthess.

Kennedy, K. J. (2008). The Citizenship Curriculum: Ideology, Content and Organization. In J. Arthur, I. Davies, \& C. Hahn (Hrsg.), The SAGE Handbook of Education for Citizenship and Democracy (S. 483-518). London: SAGE.

Krammer, R. (2008). Kompetenzen durch Politische Bildung. Ein Kompetenz-Strukturmodell. In Forum Politische Bildung (Hrsg.), Kompetenzen durch Politische Bildung, 29, 5-14.

Kuckartz, U. (2014). Qualitative Inhaltsanalyse. Methoden, Praxis, Computerunterstützung (2. Aufl.). Weinheim: Beltz Juventa.

Lipowsky, F., \& Rzejak, D. (2012). Lehrerinnen und Lehrer als Lerner - Wann gelingt der Rollentausch? Merkmale und Wirkungen wirksamer Lehrerfortbildungen. Schulpädagogik heute, 5, 1-13.

Manzel, S. (2007). Kompetenzzuwachs im Politikunterricht. Ergebnisse einer Interventionsstudie zum Kernkonzept Europa. Münster: Waxmann.

Manzel, S., \& Sowinski, M. (2014). Lernaufgaben in der politikdidaktischen Forschung Erste Ergebnisse aus einem Video-Pilot zu Unterrichtsfragen und Aufgabenstellungen bei Lehrkräften und Praktikant/-innen im Politik-/SoWi-Unterricht. In B. Ralle, S. Prediger, M. Hammann, \& M. Rothgangel (Hrsg.), Lernaufgaben entwickeln, bearbeiten und 
überprüfen - Ergebnisse und Perspektiven fachdidaktischer Forschung (S. 71-84). Münster: Waxmann.

Oberle, M. (2012). Politisches Wissen über die Europäische Union. Subjektive und objektive Politikkenntnisse von Jugendlichen. Wiesbaden: Springer VS.

Oberle, M., \& Forstmann, J. (2015a). Effekte des Fachunterrichts «Politik und Wirtschaft» auf EU-bezogene Kompetenzen von Schülerinnen und Schülern. In G. Weisseno \& C. Schelle (Hrsg.), Empirische Forschung in gesellschaftswissenschaftlichen Fachdidaktiken (S. 67-81). Wiesbaden: Springer VS.

Oberle, M., \& Forstmann, J. (2015b). Lehrerfortbildungen zur politischen EUBildung eine empirische Begleitstudie. In M. Oberle (Hrsg.), Die Europäische Union erfolgreich vermitteln (S. 193-212). Wiesbaden: Springer VS.

Petrik, A. (2010). Core concept spolitical compasss. How Kitschelt's model of liberal, socialist, libertarian and conservative orientations can fill the ideology gap in civic education. Journal of Social Science Education, 9(4), 45-62.

Rappenglück, S. (2004). Europäische Komplexität verstehen lernen. Schwalbach: Wochenschau. Reinhardt, V., Waldis, M., \& Ziegler, B. (2010). Wissen, Interesse und Einstellungen - Schweizerische Ergebnisse aus der Interventionsstudie des TEESAEC-Programms. In I. Juchler (Hrsg.), Kompetenzen in der politischen Bildung, (S. 182-195). Schwalbach: Wochenschau.

Richter, D. (2004). «Doing European` satt «Europäische Identität` als Ziel politischer Bildung. In G. Weisseno (Hrsg.), Europa verstehen lernen. Eine Aufgabe des Politikunterrichts (S. 172-184). Bonn: Bundeszentrale für politische Bildung.

Sander, W. (2009). Wissen: Basiskonzepte der Politischen Bildung. Informationen zur Politischen Bildung, 30 [onlineversion], 57-60. http://www.politischebildung.com/pdfs/30_sander. pdf [Zugriff am 19.05.2020].

Scheller, J., \& Thyroff, J. (2020, im Druck). Qualitative Unterrichtsanalyse hinsichtlich der adressierten politischen (Teil-)Kompetenzen in der Nordwestschweiz. In M. Oberle \& M.-M. Stamer (Hrsg.), Politische Bildung in internationaler Perspektivel International Perspectives on Civic Education. Frankfurt a.M.: Wochenschau.

Scheller, J., Thyroff., J., Schneider, C., \& Waldis, M. (eingereicht). Konzeptuelles Lernen in Politischer Bildung zwischen theoretischer Normierung und praktischer Realisierung. Zeitschrift für Didaktik der Gesellschaftswissenschaften, 11(2).

Spiess, C. (2014). Quellenarbeit im Geschichtsunterricht. Die empirische Rekonstruktion von Kompetenzerwerb im Umgang mit Quellen. Göttingen: V\&R unipress.

Stamm, M. (2007). Jugendliche in der Schweiz und ihr Blick nach Europa. Ausgewählte Ergebnisse einer Schweizer Langzeitstudie. In H. Biedermann, F. Oser, \& C. Quesel (Hrsg.), Vom Gelingen und Scheitern Politischer Bildung. Studien und Entwürfe (S. 383-393). Zürich: Rüegger.

Staub, F. C. (2001). Fachspezifisch-pädagogisches Coaching: Theoriebezogene Unterrichtsentwicklung zur Förderung von Unterrichtsexpertise. Beiträge zur Lehrerbildung, 19(2), 175-198.

Thyroff, J., Hedinger, F., \& Waldis, M. (2019). Menschenrechte als Gegenstand Politischer Bildung im Geschichtsunterricht. Zur Realisierung von politischen und historischen Kompetenzen in Einstiegssequenzen. Zeitschrift für Didaktik der Gesellschaftswissenschaften 10(1), 93-112.

Thyroff, J., \& Waldis, M. (2020). Politische Bildung unterrichten. Eine Reflexion über Erscheinungsformen und Herausforderungen anhand eines Fallbeispiels auf Sekundarstufe I zum Thema "Europa-EU-Schweiz». In B. Ziegler \& A. Glaser (Hrsg.), Direkte Demokratie im Kanton Aargau in Geschichte und Gegenwart (S. 69-87). Aarau: Schulthess.

Waldis, M., Hodel, J., \& Fink, N. (2012). Lernaufgaben im Geschichtsunterricht und ihr Potential zur Förderung historischer Kompetenzen. Zeitschrift für Didaktik der Gesellschaftswissenschaften, 3(1), 142-157.

Waldis, M., Schneider, C., Hedinger, F., \& Thyroff, J. (2017). Europa - eine Fallanalyse 
zur Umsetzung der politischen Perspektive im fächerübergreifenden Unterricht mit Geschichtec. In P. Mittnik (Hrsg.), Empirische Einsichten in der Politischen Bildung, herausgegeben (Österreichische Beiträge zur Geschichtsdidaktik 12, S. 39-54). Innsbruck: Studienverlag.

Weisseno, G., \& Eck, V. Hrsg. (2009). Teaching European Citizens. A Quasi-experimental Study in Six Countries. Münster: Waxmann.

Wyss, C., \& Schneider, C. (2012). Interesse und Motivation als Voraussetzung für Wissensaufbau - Befragung von Schülerinnen und Schülern zum WebQuest und zu EU und Europa. B. Ziegler \& D. Allenspach (Hrsg.), Was Schweizer Jugendliche von der EU wissen - Die schweizerische TEESAEC-Studie (S. 55-74). Zürich: Rüegger.

Zhang, T., Torney-Purta, J., \& Barber, Carolyn E. (2012). Student's Conceptual Knowledge and Process Skills in Civic Education: Identifying Cognitive Profiles and Classroom Correlates. Theory \& Research in Social Education, 40(1). doi:10.1080/00933104.2012.649467

Ziegler, B., \& Reinhardt, V. (Hrsg.). (2012). Was Schweizer Jugendliche von der EU wissenDie schweizerische TEESAEC-Studie. Zürich: Rüegger.

Schlagworte: Politikunterricht, politische Fachlichkeit, politische Kompetenzen, Unterrichtsvideographie, Inhaltsanalyse

\section{Citoyenneté au cycle 3 . Aspects et défis de séquences d'enseignement sur "Europe - UE - Suisse»}

\section{Résumé}

Avec l'introduction du curriculum 21, les professeurs d'histoire seront confrontés au défi d'enseigner également l'éducation politique au cycle 3 - une matière qui, cependant, dispose de ses propres principes, perspectives, concepts et compétences. Dans quelle mesure ces professeurs parviennent-ils aujourd'hui à enseigner cette matière véritablement politique ? Dans ce texte, nous présentons les résultats de nos analyses des pratiques d'enseignement, que nous avons obtenues grâce à huit doubles leçons sur le thème «Europe - UE - Suisse», menées par quatre enseignants. Nous nous concentrons sur deux aspects. Dans quelle mesure les enseignants a) affichent-ils une expertise politique au niveau de la matière et b) abordent-ils les compétences politiques dans les tâches formulées?

Mots clés: Citoyenneté, professionnalisme politique, compétences politiques, enseignement de la vidéographie, analyse de contenu

\section{Educazione civica nel $3^{\circ}$ ciclo. Aspetti e sfide sull'esempio delle sequenze didattiche "Europa - UE - Svizzera»}

\section{Riassunto}

Con l'introduzione del curriculum 21, gli insegnanti di storia si troveranno ad affrontare la sfida di insegnare anche l'educazione civica nel $3^{\circ}$ ciclo - una materia che, tuttavia, ha i propri principi, le sue prospettive, i suoi concetti e le sue competenze. In che misura gli insegnanti riescono attualmente a realizzare pienamente 
questa materia, autenticamente politica? Nel testo presentiamo i risultati delle nostre analisi didattiche che abbiamo ottenuto attraverso otto lezioni doppie sul tema "Europa - UE - Svizzera», condotte da quattro insegnanti. Ci concentriamo su due aspetti: In che misura gli insegnanti a) si rendono conto delle competenze politiche necessarie a livello di materia e b) affrontano le competenze politiche nei compiti formulati?

Parole chiave: Educazione civica, professionalità politica, competenze politiche, insegnamento della videografia, analisi dei contenuti 


\section{Civic education at lower secondary level. Manifestations and challenges with the example of teaching sequences on "Europe - EU - Switzerland»}

\section{Summary}

With the introduction of curriculum 21, history teachers at lower secondary level will be faced with the challenge of also teaching civic education - a subject that, however, has its own principles, perspectives, concepts and competencies. To what extent do teachers currently succeed in achieving this? We present the results of our teaching analyses, based on eight double lessons on the subject of «Europe - EU - Switzerland», carried out by four teachers. We focus on two aspects: To what extent are the teachers a) displaying political expertise on the subject level and b) addressing subject specific competences in the formulated assignments?

Keywords: Civic education, subject specific perspectives, subject specific competences, video study, content analysis

\section{Autoren}

Zentrum Politische Bildung und Geschichtsdidaktik der PH FHNW, Villa Blumenhalde, Küttigerstrasse 21, CH-5000 Aarau

Julia Thyroff, Dr. des., ist Mitarbeiterin am Zentrum Politische Bildung und Geschichtsdidaktik der PH FHNW und dort u. a. in der Unterrichtsforschung in Politischer Bildung tätig. Weiterhin ist er Geschichtsdidaktiker an der Universität Greifswald (Deutschland).

E-Mail: julia.thyroff@fhnw.ch

Jan Scheller ist wissenschaftlicher Assistent am Zentrum Politische Bildung und Geschichtsdidaktik der PH FHNW und dort u. a. in der Unterrichtsforschung in Politischer Bildung tätig.

E-Mail: jan.scheller@fhnw.ch

Claudia Schneider war bis Juni 2019 Mitarbeiterin am Zentrum Politische Bildung und Geschichtsdidaktik der PH FHNW und in der Entwicklung von Lehr-/Lernmaterialien sowie Forschung und Lehre zu Politischer Bildung tätig. E-Mail: claudia.schneider@fhnw.ch

Monika Waldis, Prof. Dr., ist Leiterin des Zentrums Politische Bildung und Geschichtsdidaktik der PH FHNW und führt geschichts- und politikdidaktische Forschungs- und Entwicklungsprojekte durch.

E-Mail: monika.waldis@fhnw.ch 